NBER WORKING PAPERS SERIES

\title{
STOCHASTIC DEVALUATION RISK AND THE EMPIRICAL FIT OF TARGET ZONE MODELS
}

Giuseppe Bertola

Lars E.O. Svensson

Working Paper No. 3576

NATIONAL BUREAU OF ECONOMIC RESEARCH

1050 Massachusetts Avenue

Cambridge, MA 02138

January 1991

\footnotetext{
Part of the work for this paper was done while Giuseppe Bertola visited the IIES; another part was done while Lars Svensson visited the Research Department at IMF. Both authors thank their respective host institutions for their hospitality. We are grateful to Bernard Dumas, Robert Flood, Harald Lang and Andrew Rose for helpful conversations, and to seminar participants at IIES for their comments. We alone are responsible for any errors. This paper is part of NBER's research program in International studies. Any opinions expressed are those of the authors and not those of the National Bureau of Economic Research.
} 
NBER Working Paper \#3576

January 1991

STOCHASTIC DEVALUATION RISK AND THE
EMPIRICAL FIT OF TARGET ZONE MODELS

\section{ABSTRACT}

This paper proposes a tractable and realistic nonlinear model of exchange rate dynamics, and argues that its predictions are consistent with available empirical evidence on exchange rate and interest differential behavior in real-life target zones. In our model, the exchange rate fluctuates between given boundaries for random lengths of time and jumps discretely when devaluations occur. We allow for stochastic variability in the likelihood and size of devaluations, and we provide explicit solutions for the stochastic processes followed by the exchange rate and by the expected rate of depreciation. The model produces realistic patterns of covariation between exchange rates and interest rate differentials, and provides interesting interpretations of available empirical evidence. We also specify how to infer devaluation risk from target zone data.

Giuseppe Bertola Department of Economics Fisher Hall Princeton University Princeton NJ 08544
Lars E.O. Svensson Institute for International Economic studies s-10691 stockholm SWEDEN 


\section{Introduction}

Under reasonable assumptions, most of the available target zone models of exchange rate dynamics imply a deterministic, nonlinear relationship between the exchange rate's deviation from its central parity on the one hand, and interest rate differentials and "fundamentals" (i.e. variables appearing in excess money demand functions, such as liquidity and activity levels) on the other. These predictions have received only limited validation from empirical work. Svensson (1990b) finds Swedish data to be qualitatively consistent with his model, in which the target zone is defended by infinitesimal interventions and devaluations of fixed size occur with constant probability intensity; the fit between theory and data is far from perfect, however, and a strongly serially correlated component is left unexplained in the relationship between exchange rates and interest rate differentials. ${ }^{1}$ The model of Bertola and Caballero (1990b) has different empirical implications. which are consistent with German, French, and Italian exchange and interest rate data: but empirical support for the predicted relationship between interest rate differentials and exchange rate fluctuations is again only qualitative, and rather weak. Using nonparametric methods on a variety of exchange rate, interest rate, and "fundamental" data, Meese and Rose (1990) and Flood, Rose and Mathieson (1990) do not find any evidence of significant nonlinearities: the components left unexplained by nonlinear models are highly serially correlated and, in many cases, so large as to raise serious doubts on the empirical validity of target-zone models.

More general theoretical frameworks could, of course, provide an explanation for the disappointing empirical performance of the first generation of target zone models. In general, what is necessary to reconcile theory and evidence is allowance for a source of exchange rate and interest rate fluctuation other than traditional fundamentals. For the theoretical nonlinearities to become apparent in empirical work, it would then be necessary to control for the time variation of state variables other than the exchange rate. Of course, the newly introduced state variables should ultimately be given an economically meaningful interpretation. Bertola and Caballero (1990a), for example, argue that repeated interventions should affect the likelihood and/or the size of realignments, and propose stylized models in which the level of reserves affects the position and shape of the relationship between expected depreciation and exchange rates.

In this paper, we extend the model of Svensson (1990b) to allow for stochastic fluctuations in the size and/or likelibood of devaluations. We do not provide a specific model

1 Lehmussaari and Suvanto (1990) provide similar evidence from Finnish data. 
of what the economic determinants of such fluctuations might be. Still, the probabilistic structure we propose is flexible enough to generate realistic patterns of covariation between observables: in our model, exchange rates and interest rate differentials are influenced by the current rate of expected devaluation on the one hand, and by the character of the stochastic process it follows over time on the other, suggesting potentially fruitful avenues for further empirical and theoretical work.

Section II introduces our basic assumptions and derives the equilibrium relationship between exchange rates, fundamentals, and the expected rate of devaluation. Section III studies the instantaneous interest rate differential and the term structure of interest rate differentials. Section IV discusses the results, emphasizing their implications for the interpretation of existing evidence and for further empirical work. Section V specifies how the expected rate of devaluation can be inferred from data on exchange rates and interest rate differentials. Section VI concludes and outlines possible theoretical and empirical extensions of our work.

\section{The exchange rate}

We denote with $x(t)$ the logarithm of the exchange rate at time $t$, measured as units of home currency per unit foreign currency (or per unit of a basket of foreign currencies). We follow the literature in assuming that it satisfies the relationship

$$
x(t)=f(t)+\alpha E_{t}[d x(t)] / d t,
$$

where the scalar $f(t)$ denotes the fundamental determinants of the exchange rate, and $\alpha$ indexes the extent to which the exchange rate level depends on its own expected rate of change in continuous time. We also follow the literature in assuming that $f(t)$ can be modeled as an exogenously given stochastic process which is compatible with maintaining the exchange rate within a prespecified range $\left(x^{l}, x^{u}\right)$ unless a realignment occurs. For concreteness, we refer to all realignments as devaluations, with devaluations of negative size representing revaluations.

The models that have inspired the empirical work reviewed in the Introduction assume that the probability distribution of future interventions and devaluations is fully determined (for given parameters) by the current level of $f$. Thus, the $x(t)$ and $E_{t}\{d x\} / d t$ sample paths are uniquely determined by the sample path of $\{f(t)\}$, and equation (2.1) implies a deterministic (nonlinear) relationship between the position of $x(t)$ relative to the limits of its current fluctuation band and its expected rate of change. ${ }^{2}$ The empirical

2 The shape of the relationship, of course, depends on the specific structure of the model 
evidence reviewed above, however, suggests that expected depreciation rates should also be allowed to fluctuate independently of the exchange rate's position in the band. We proceed to do so in the context of the Svensson (1990b) model of a target zone subject to realignments.

We assume $\{f(t)\}$ to follow a process with stochastic differential

$$
d f(t)=\mu_{f} d t+\sigma_{f} d W_{f}(t)+d L(t)-d U(t)+d c(t) .
$$

Here, $\mu_{f}$ and $\sigma_{f}$ are scalar constants; $\left\{W_{f}(t)\right\}$ is a standard Wiener process; and $\{L(t)\}$, $\{U(t)\}$ are nondecreasing, continuous processes which increase only when the exchange rate is (respectively) at the lower or upper boundary of its current fluctuation band. The infinitesimal increments of these two regulator processes are those necessary to maintain the exchange rate within the boundaries of its current fluctuation band. The process $\{c(t)\}$ is the (log of) central parity, which is a jump process; it jumps at realignments and is constant between realignments.

It will be convenient to write

$$
x(t)=\tilde{x}(t)+c(t), \quad f(t)=\tilde{f}(t)+c(t),
$$

where $\tilde{x}(t) \equiv x(t)-c(t)$ denotes the exchange rate's log-deviation from central parity, and $\tilde{f}(t) \equiv f(t)-c(t)$ denotes the fundamental's deviation from central parity. When a devaluation occurs, the upper and lower boundaries of the exchange rate fluctuation band are redefined and both the central parity, the exchange rate and the fundamental undergo a discrete change. We denote with $z(t)$ the size of the exchange rate jump if a devaluation occurs at time $t$. In what follows, it will often be convenient to assume that $z(t)=c\left(t_{+}\right)-c\left(t_{-}\right)$, i.e. that the exchange rate's position within the band is unchanged by a realignment; in general, however, this need not be the case.

considered. If devaluations never occur, or if their likelihood and size is independent of the exchange rate's position in the target zone (like in Svensson (1990b)), then the expected rate of depreciation must be lower the closer is the exchange rate to its current upper bound: quite intuitively, a more depreciated exchange rate is less likely to depreciate further than to appreciate. If realignments only occur when the exchange rate is at one of the target zone's boundaries, conversely, the expected rate of depreciation can be an increasing function of the exchange rate's distance from its current lower bound (see Miller and Weller (1989) and Bertola and Caballero (1990b)). 
We let a devaluation resulting in an exchange rate jump of random size $z(t)$ occur with probability $\nu(t) d t$ in a time interval of infinitesimal length $d t$, while no devaluation occurs with probability $1-\nu(t) d t$. Because risk neutrality and instantaneous equilibrium are assumed in equation (2.1), only the expected size of the exchange rate jump associated to a realignment in the next instant has a bearing on exchange rate determination; the higher moments of the size of devaluations, and expectations of devaluation over longer horizons, are irrelevant. ${ }^{3}$ We therefore define an expected rate of devaluation process $\{g(t)\}$ as

$$
g(t) \equiv \frac{1}{d t}\left(\nu(t) E_{t}[z(t)] d t\right)=\nu(t) \bar{z}(t)
$$

where $\bar{z}(t) \equiv E_{t}[z(t)]$.

If either or both of $\bar{z}(t)$ and $\nu(t)$ fluctuate stochastically over time, so does $g(t)$. We assume $\{g(t)\}$ to follow a Brownian motion process, allowing a possibly nonzero correlation between its increments and those driving the fundamentals process:

$$
d g(t)=\mu_{g} d t+\sigma_{g} d W_{g}(t), \quad d W_{g}(t) d W_{f}(t)=\rho d t, \quad|\rho| \leq 1 .
$$

The vector process $\{f(t), g(t)\}$ is Markov in levels: ruling out bubbles, both the exchange rate and the expected rate of depreciation can then be written, for given parameters and given target zone boundaries, as functions of the two state variables $f$ and $g$.

The presence of multiple state variables would typically make it very difficult or even impossible to solve a nonlinear forward-looking model. 4 Fortunately, however, equation (2.1) and the presence of "target zone" limits to exchange rate fluctuations impose strong

3 For clarity of interpretation, we express our solution in terms of limits to exchange rate fluctuation and in terms of exchange rate jumps at devaluation times. As long as exchange rate bubbles are ruled out, so that (2.1) can be integrated forward to yield $x(t)=\int_{t}^{\infty} e^{-(\tau-t) / \alpha} E_{t}[f(\tau)] d \tau / \alpha$, the model implies limits to fundamertal fluctuations, and jumps as well as continuous changes in (the probability distribution of) fundamentals. While this relationship can remain implicit in this section, it will be necessary to be more specific when considering finite-maturity interest rate differentials in Section III.

- Using the multivariate form of Ito's differentiation rule, $x(f, g)$ could be written in terms of the parameters appearing in the stochastic differentials (2.2) and (2.4) and of the partial derivatives of the $x(f, g)$ function. The resulting partial differential equation for $x(f, g)$ would then need to be solved jointly with appropriate boundary conditions at the margins of the exchange rate fluctuation band; namely, the first-order derivatives 
restrictions on the joint probability distribution of exchange rates and expected depreciation rates and, consequently, on the shape of the $x(.,$.$) function. In the model we are$ considering, the expected depreciation rate is the sum of two components: the expected depreciation rate within the band, denoted $E_{t}[d \tilde{x}] / d t$, and the expected rate of devaluation. g. Thus, we can write

$$
\frac{E_{t}[d x]}{d t}=\frac{E_{t}[d \tilde{x}]}{d t}+g .
$$

Substitution of (2.5) into (2.1), together with (2.3), allows us to write

$$
x(f, g)=f+\alpha g+\alpha \frac{E_{\ell}[d \tilde{x}]}{d t}=\tilde{f}+\alpha g+\alpha \frac{E_{t}[d \tilde{x}]}{d t}+c=\tilde{x}(\tilde{f}, g)+c
$$

where $\tilde{x}$ hence depend only on $\tilde{f}$ and $g$. It is not difficult to see that all $\tilde{f}, g$ pairs such that $\tilde{f}+\alpha g$ is constant must be consistent with the some exchange rate level within the band. We then define the new state variable

$$
h(t) \equiv \tilde{f}(t)+\alpha g(t)
$$

and note that, under our assumptions, $\{h(t)\}$ is a Brownian motion process with differential

$$
\begin{aligned}
& d h(t)=\mu d t+\sigma d W(t), \text { where } \\
& \mu \equiv \mu_{f}+\alpha \mu_{g} \quad \text { and } \sigma \equiv \sqrt{\sigma_{f}^{2}+\alpha^{2} \sigma_{g}^{2}+2 \alpha \rho \sigma_{f} \sigma_{g}} .
\end{aligned}
$$

Thus, exchange rate determination is reduced to a single state variable problem, the solution $\tilde{x}(h)$ of the equation

$$
\tilde{x}(h)=h+\alpha \frac{E_{t}[d \tilde{x}]}{d t}
$$

with boundary conditions

$$
\tilde{x}\left(h^{\prime}\right)=x^{\prime}-c, \quad \tilde{x}\left(h^{u}\right)=x^{u}-c, \quad \tilde{x}^{\prime}\left(h^{u}\right)=\tilde{x}^{\prime}\left(h^{\prime}\right)=0,
$$

where $h^{\prime}, h^{\mu}$ are defined as the points in the state space where the exchange rate reaches its current lower and (respectively) upper boundaries.

should equal zero at the margins of the fluctuation band, where - since the regulators are applied with probability one- the expected rate of depreciation must be the same regardless of whether or not the regulators are operating. This approach would encounter nontrivial technical difficulties, however, because few uniqueness and representation results are available for multivariate boundary problems of this type. 
The solution is fully analogous to that appropriate for a credible target zone (see Krugman (1990) and Froot and Obstfeld (1989)):

$$
\tilde{x}(h)=h+\alpha \mu+A_{1} e^{\lambda_{1} h}+A_{2} e^{\lambda_{2} h}
$$

where $\lambda_{1}$ and $\lambda_{2}$ are the roots of the characteristic equation $\frac{1}{2} \alpha \sigma^{2} \lambda^{2}+\alpha \mu \lambda-1=0$ :

$$
\lambda_{1}=-\frac{\mu}{\sigma^{2}}+\sqrt{\frac{\mu^{2}}{\sigma^{4}}+\frac{2}{\alpha \sigma^{2}}}, \quad \lambda_{2}=-\frac{\mu}{\sigma^{2}}-\sqrt{\frac{\mu^{2}}{\sigma^{4}}+\frac{2}{\alpha \sigma^{2}}} .
$$

The constants $A_{1}, A_{2}$ are chosen, as a function of the currently enforced exchange rate boundaries, to satisfy the boundary conditions:

$$
\begin{array}{cc}
h^{u}+\alpha \mu+A_{1} e^{\lambda_{1} h^{u}}+A_{2} e^{\lambda_{2} h^{u}}=x^{u}-c, & 1+A_{1} \lambda_{1} e^{\lambda_{1} h^{u}}+A_{2} \lambda_{2} e^{\lambda_{2} h^{u}}=0, \\
h^{l}+\alpha \mu+A_{1} e^{\lambda_{1} h^{l}}+A_{2} e^{\lambda_{2} h^{\prime}}=x^{l}-c, & 1+A_{1} \lambda_{1} e^{\lambda_{1} h^{l}}+A_{2} \lambda_{2} e^{\lambda_{2} h^{\prime}}=0 .
\end{array}
$$

Figure 2.1 portrays the familiar S-shaped relationship between $h$ and $\tilde{x}$. The parameters are $x^{l}-c=-1.5$ percent, $x^{u}-c=1.5$ per cent (as in the Swedish exchange rate band); if time is measured in years, $\alpha=1$ year, $\mu=2$ per cent per year, and $\sigma=5$ per cent per $\sqrt{\text { year. }}{ }^{5}$ We shall use these parameters throughout the paper.

The solution can equivalently be written in terms of the two driving processes $\tilde{f}$ and $g$ as

$$
\tilde{x}(\tilde{f}, g)=\tilde{x}(\tilde{f}+\alpha g)=\tilde{f}+\alpha g+\alpha\left(\mu_{f}+\alpha \mu_{g}\right)+A_{1} e^{\lambda_{1}(j+\alpha g)}+A_{2} e^{\lambda_{2}(\bar{f}+\alpha g)},
$$

and it is easily interpreted. Its linear part corresponds to the free-floating, bubble-free exchange rate solution that would be appropriate if fundamentals followed a mixed Brownianjump process, with the product of the probability intensity and expected size of jumps following, in turn, the Brownian process of equation (2.4). In such an environment, the expected rate of depreciation would always be equal to $g+\mu_{f}+\alpha \mu_{g}$, the last term reflecting the fact that every change in the likelihood and/or size of jumps is immediately reflected in the level of $\tilde{x}$ via the no-arbitrage condition (2.1) if $\alpha \neq 0$, and that the expected rate of devaluation is expected to increase or decrease at rate $\mu_{g}$. The exponential terms, in turn, reflect the stabilizing expectations due to knowledge that the infinitesimal regulators will be activated when and if the $h$ process fluctuates to either boundary of its current fluctuation zone. As usual, the constant $A_{1}$ associated to the positive $\lambda_{1}$ root is negative,

3 The dimensionality of $\alpha$ and $\sigma$ follows from noting that $E_{t}[d x / d t]$ in (2.1) has the dimension of time and $\sigma^{2}$ has the dimension of $1 /$ time. 
to satisfy smooth pasting: thus, a (say) upward movement of $h$, which may be due to any one of many combination of $\tilde{f}$ and $g$ movements, makes it more (less) likely that the upper (lower) regulator will be activated in the near future, and forward-looking agents recognize that such regulation induces a downward bias in the expected discounted integral of future fundamentals. Figure 2.2 shows the relation between the exchange rate and $\tilde{f}$, for given $g$ : the curve on the right corresponds to $g=0, \hat{f}=h$, while the curve on the left corresponds to a positive $g$; the horizontal distance between the two curves is $\alpha g$.

Figure 2.3 displays a three-dimensional plot of the relationship between $\tilde{x}, \bar{f}$, and $g$. The function $\tilde{x}(\tilde{f}, g)$ is defined on the set $\left\{\tilde{f}, g \mid h^{l} \leq \tilde{f}+\alpha g \leq h^{u}\right\}$, namely the region between the flat southwest section and the flat northeast section of the surface in the Figure. The points in $\tilde{f}, g$ space that yield the same exchange rate lie on parallel lines with slope $d \tilde{f} / d g=-\alpha$. We note that, in addition to the $S$-shapes in the $\tilde{f}$ direction for fixed $g$, the surface's sections describe $S$-shapes in the $g$ direction for fixed $\tilde{f}$.

Several features of the solution deserve to be mentioned. Krugman (1990) notes that stabilizing expectations of intervention yield an $\tilde{x}(\tilde{f})$ function that is everywhere flatter than the $45^{\circ}$ line that would apply in a free float, thus allowing the fundamental band to be wider than the exchange rate band (the "honeymoon effect"). In terms of this interpretation, the range of allowable fundamental values is shifted by the possibility of devaluations: the larger is $g$, the smaller is the upper bound on $\vec{f}$ (consider Figure 2.2 again, where different devaluation risks cause the $S$ shape to shift sideways for given exchange rate bands). More interestingly, the shape of the $S$ and the width of the fluctuation band are affected, for given $\left(x^{l}, x^{u}\right)$, by the parameters of the stochastic process followed by $\{g(t)\}$. If $\sigma_{g}>0$ and $\rho \geq 0$, for example, then $\sigma>\sigma_{f}$ and the relationship between exchange rates and fundamentals is flatter, for given $g$, than it would be if $g$ were constant as in Svensson (1990b). If $g$ could be taken as given, this would imply that fundamentals can deviate by larger amounts before "intervention" is called for. Since fluctuations in $\tilde{f}$ occur concurrently with fluctuations in $g$, however, intervention in general has to occur more often and by larger amounts, to offset shifting expectations of devaluation as well as fluctuations in $\tilde{f}$ (which, following Krugman (1990) and Froot and Obstfeld (1989), may be taken to represent random fluctuations in the velocity of circulation of money, in activity levels, and so on).

\section{The interest rate differentials}

In this section we shall derive the implications of stochastic devaluation risk for the instantaneous interest rate differentials and for the term structure of interest rate differentials 
(the differential yield curve). In particular, we shall study the determinant of the correlation between interest rate differentials and exchange rates, with a view to uncovering ways in which devaluation risks and their behavior over time might be inferred from data on interest rate differentials and exchange rates (see Section IV below).

Let $\delta(t ; \tau)$ denote the interest rate differential at time $t$ between default-free discount bonds that mature at time $t+\tau$ and are denominated, respectively, in home and foreign currency (or a basket of foreign currencies). Uncovered interest rate parity should hold if agents are risk neutral or (approximately) if the risk premium is small. ${ }^{6}$ For a finite maturity $\tau>0$ we then use the approximation

$$
\delta(t ; \tau)=\frac{E_{t}[x(t+\tau)]-x(t)}{\tau}:
$$

the interest rate differential equals the expected depreciation to maturity, divided by the maturity.

\section{Instantaneous interest rate differentials}

If we let the maturity approach zero, we get the instantaneous interest rate differential,

$$
\delta(t ; 0)=E_{t}[d x] / d t
$$

which equals the expected instantaneous rate of depreciation. Using (2.1) and (2.10), we see that the instantaneous interest rate differential can be written as a function of $h$ and $g$,

$$
\delta(h, g ; 0)=\tilde{\delta}(h ; 0)+g, \text { for } h^{l} \leq h \leq h^{4},
$$

where

$$
\tilde{\delta}(h ; 0)=\frac{1}{d t} E_{t}[d \tilde{x}]=\mu+\left(A_{1} e^{\lambda_{1} h}+A_{2} e^{\lambda_{2} h}\right) / \alpha=(\bar{x}(h)-h) / \alpha
$$

is the expected (instantaneous) depreciation within the exchange rate band, the last equality following from (2.9). This term is the interest rate differential for a zero expected rate of devaluation, and is extensively examined in Svensson (1989). It depends only on the state variable $h$, with $\delta^{\prime}(h)<0$; since the relation between $h$ and the exchange rate is monotonic, the expected rate of depreciation within the band is also a decreasing function of the exchange rate: quite intuitively, the larger the value of the exchange rate in the band, the larger is the probability of an intervention in the near future to (e.g.) reduce

- Svensson (1990a) shows that the risk premium is likely to be small for narrow target zones and moderate devaluation risks. 
money supply and appreciate the exchange rate, and the smaller is the expected rate of depreciation.

In (3.3a), the expected (instantaneous) rate of devaluation is added to the expected rate of depreciation within the band. As shown if Figure 3.1, this simply shifts the instantaneous interest rate differential up by $g$ when it is plotted as a function of the exchange rate (or of the state variable $h$ ). ${ }^{7}$ We understand from Figure 3.1 that as both fundamentals and the expected rate of depreciation fluctuate overtime, almost any pattern of exchange rates and instantaneous interest rate differential observations can result. In this section we focus on the correlation between the instantaneous interest rate differential and the exchange rate, postponing further discussion of possible patterns to Section IV below.

If the relative variability of the expected rate of devaluation $g$ is low, most of the variability of the state variable $h$ (and hence of the exchange rate) is due to variations in the fundamental $\tilde{f}$ : then the curve $\delta(\tilde{x}, g ; 0)$ in Figure 3.1 does not shift much, observations of exchange rates and interest rate differentials all lie along it, and the instantaneous correlation between exchange rates and interest rate differentials is negative. If instead the relative variability of the expected rate of devaluation is high, so that most of the variability of the state variable $h$ (and hence of the exchange rate) is due to variations in $g$, then the curve $\delta(\bar{x}, g ; 0)$ in Figure 3.1 will shift around a lot. Consider an increase in $g$ : this will increase the exchange rate, and would move the observation down and to the right if the curve $\delta(\tilde{\boldsymbol{x}}, g ; 0)$ did not shift. But the curve shifts up with the increase in $g$, and this moves the observation up. Clearly, the correlation between exchange rates and interest rate differentials will be less negative, and perhaps positive.

These graphical arguments can be formalized computing the instantaneous covariance between the exchange rate and the instantaneous interest rate differential, denoted $\sigma_{x} \delta$ :

$$
\sigma_{x \delta}=\left(\left(\sigma_{f}^{2}+\alpha^{2} \sigma_{g}^{2}+2 \alpha \rho \sigma_{f} \sigma_{g}\right) \tilde{\delta}^{\prime}(h)+\rho \sigma_{f} \sigma_{g}+\alpha \sigma_{g}^{2}\right) \tilde{x}^{\prime}(h) .
$$

If $\sigma_{g}=0$, this reduces to $\sigma_{x} \delta=\sigma_{f}^{2} \tilde{x}^{\prime}(h) \tilde{\delta}^{\prime}(h)<0$. Conversely, if $\sigma_{f}=0$ we have $\sigma_{x \delta}=\alpha\left(\alpha \tilde{\delta}^{\prime}(h)+1\right) \tilde{x}^{\prime}(h) \sigma_{g}^{2}=\alpha \sigma_{g}^{2}\left(\tilde{x}^{\prime}(h)\right)^{2}>0$.

These results may explain some apparent inconsistencies in the empirical literature on target zones: Svensson (1990b) finds a negative correlation between exchange rates and interest rate differentials for the Swedish target zone. Lehmussaari and Suvanto (1990) also

7 If we plot the instantaneous interest rate differential as a function of the fundamental $\tilde{f}$, a larger $g$ shifts the relationship to the left as well as upwards, since the band for $\tilde{f}$ is shifted to the left when $g$ increases. 
find a negative correlation for the Finnish target zone. In contrast, Bertola and Caballero (1990b) find a positive correlation between exchange rates and interest rate differentials for France and Italy in ERM, the exchange rate mechanism within EMS. Flood, Rose and Mathieson (1990) document both negative and positive correlations in different subperiods for different countries in ERM. One possible explanation of these conflicting results is that the relative variability of the expected rate of devaluation has been low in Sweden and Finland for the periods examined, whereas the relative variability of the expected rate of devaluation has varied between countries and subperiods in ERM. ${ }^{8}$

In Svensson (1989) a direct negative relation is derived between the instantaneous standard deviations of the instantaneous interest rate differential and the exchange rate,

$$
\sigma_{z}+\alpha \sigma_{\delta}=\sigma_{f}
$$

With stochastic devaluation risk, the corresponding relation can be written

$$
\sigma_{\delta}=\frac{1}{\alpha} \sqrt{\left(\sigma-\sigma_{x}\right)^{2}-2 \alpha(\rho+\alpha)\left(\sigma-\sigma_{x}\right) \sigma_{g}+\sigma_{g}^{2}} .
$$

We see that while the direct negative relation holds if $\sigma_{g}=0$, the relation between $\sigma_{x}$ and $\sigma_{\delta}$ need not be negative in general. The derivative $d \sigma_{\delta} / d \sigma_{x}$ can be expressed as

$$
\frac{d \sigma_{\delta}}{d \sigma_{x}}=-\frac{\sigma_{\delta}-(\rho+\alpha) \sigma_{g}}{\alpha \sigma_{\delta}}
$$

where $\sigma_{\bar{\delta}}$ denotes the instantaneous standard deviation of $\tilde{\delta}(h ; 0)$ (and fulfills $\sigma_{\bar{\delta}}=(\sigma-$ $\left.\sigma_{x}\right) / \alpha$ ). Clearly $d \sigma_{\delta} / d \sigma_{x} \gtrless 0$ as $\sigma_{\dot{\delta}} \gtrless(\rho+\alpha) \sigma_{g}$. This might explain why Flood, Rose and Mathieson (1990) do not find the direct negative relation in the data.

\section{Finite-maturity interest rate differentials}

Since only the expected instantaneous rate of devaluation matters for the instantaneous interest rate differential, it has so far been unnecessary to specify exactly what happens upon devaluation. Turning to finite-maturity interest rate differentials, we need to be more specific about what happens to the expected rate of devaluation in the future.

- In the Bertola and Caballero (1990b) model, devaluations are assumed to occur only when the exchange rate is at the margin of its current fluctuation band, as is realistic for the French Franc/Deutsche Mark exchange rate. Thus, the expected rate of devaluation does vary over time: it is larger when the exchange rate approaches the upper limit of the band, and this yields an upward-sloping (albeit deterministic) relationship between interest rate differentials and exchange rates. 
What is assumed in this respect also has implications for the behavior over time of instantaneous interest rate differentials. If we maintain the assumption that the expected rate of devaluation is a Brownian motion constant parameters across devaluations, the instantaneous interest rate differentials are nonstationary. Flood, Rose and Mathieson (1990) do find some evidence of nonstationarity; but the feature is somewhat unappealing on theoretical grounds. Further, a positive drift of the expected rate of devaluation, which may be realistic over a limited period, implies an even less appealing ever growing expected rate of devaluation. It might be argued that the occurrence of a devaluation makes the target zone no more credible; but if the devaluation gives the authorities a chance to restock reserves, or restores competitiveness at least temporarily, the need for further devaluations may be less urgent, at least for a while.

In order to allow a fairly rich set of theoretical possibilities and to facilitate comparison with the empirical literature, we shall then examine three different combinations of assumptions with different implications for the stochastic properties of instantaneous interest rate differentials over extended periods of time on the one hand, and for the shape of the interest rate differential yield curves on the other:

Assumption 1: The expected rate of devaluation always follows the Brownian process (2.4), with no changes across devaluations.

Assumption 2: The expected rate of devaluation follows the Brownian process (2.4) between devaluations, but it is reset to a value drawn from a distribution with mean $\bar{g}$ when a devaluation occurs. The drift $\mu_{g}$ is also reset and drawn from a distribution with mean zero. In addition, the mean size of a devaluation $\bar{z}$ is constant; hence the probability intensity $\nu(t)=g(t) / \bar{z}$ is stochastic.

Assumption 9: Same as Assumption 2, except that the probability intensity $\nu$ is a constant, $\bar{\nu}$; hence the mean size of a devaluation $\bar{z}(t)=g(t) / \bar{\nu}$ is stochastic and, when a devaluation occurs, is reset and drawn from a distribution with mean $\overline{\bar{z}}=\bar{g} / \bar{\nu}$.

Throughout the discussion of finite-maturity interest rate differentials we shall also assume that $z(t)=c\left(t_{+}\right)-c\left(t_{-}\right)$, i.e. that immediately after a devaluation the exchange rate is in the same position within the band as immediately before the devaluation, as in the Svensson (1990b) model. Like the details of Assumptions 1-3, this is irrelevant to the determination of exchange rates and instantaneous interest rate differentials: ${ }^{2}$ it is very convenient, however, in that it makes it possible to separate the expected depreciation up

${ }^{2}$ In the numerical simulations reported in Section IV, which only concern instantaneous interest rate differentials, we in fact adopt the perhaps more realistic assumption that 
to maturity into the expected depreciation within the band absent any devaluation on the one hand, and expected shifts in the band due to devaluations on the other. Namely, the finite-maturity interest rate differentials can be written

$$
\delta(h, g ; \tau)=\tilde{\delta}(h ; \tau)+D(g ; \tau) .
$$

Denoting with $\xi(h ; \tau)$ the expected exchange rate at maturity time $t+\tau$, conditional upon the state variable being $h$ at time $t$ and conditional upon no devaluation occurring up to maturity, the first term on the right-hand side of (3.5) is

$$
\tilde{\delta}(h ; \tau) \equiv \frac{\xi(h ; \tau)-\tilde{x}(h)}{\tau}, \quad \tau>0,
$$

the interest rate differential resulting solely from exchange rate movements inside the band absent any devaluation. It depends only on $h$ and not separately on $g$. The expected future exchange rate within the band $\xi(h ; \tau)$ and the corresponding interest rate differential $\tilde{\delta}(h ; \tau)$ are computed and extensively examined in Svensson (1990b), to which paper we refer for details. Since the exchange rate is monotonic in $h$, we can write $\tilde{\delta}(\tilde{x} ; \tau)$ : in Figure 3.2 we plot this function in $\bar{x}, \delta$ space for different values of $\tau$, and in Figure 3.3 we plot it in $\tau, \delta$ space for different values of $\tilde{x} .{ }^{10}$ For each maturity, the interest rate differential is decreasing in the exchange rate, although less so for larger maturities. When maturity approaches infinity the interest rate differential approaches zero and becomes flat in Figure 3.2. For infinite maturity this is easily seen from (3.6a), since the numerator is bounded and divided by an unbounded denominator. For a finite maturity we have the complication that the expected future exchange rate $\xi(h ; \tau)$ depends on the maturity; however, the effect of dividing by the maturity can be shown to dominate and to make the interest rate differential less responsive to the exchange rate the longer the maturity.

The second term in (3.5),

$$
D(g ; \tau)=\frac{E\left[\int_{0}^{\tau} g(t) d t \mid g(0)=g\right]}{\tau}, \quad \tau>0,
$$

is the expected cumulative devaluation up to maturity, divided by maturity. We shall refer to it as the expected devaluation per unit maturity. Of course, $D(g ; 0)=g$, in accordance with the discussion of instantaneous interest rate differentials above. For $\tau>0$,

the exchange rate is set at the strong (weak) edge in the band after each devaluation (revaluation).

10 The parameters are those mentioned in Section II. The function is not symmetric around $x=0$ since $\mu$ is not zero. 
the expected devaluation per unit maturity (as a function of the current expected rate of devaluation $g$ and of the maturity $\tau$ ) depends on the drift of the stochastic process followed by the expected rate of devaluation, as well as on what is assumed to occur to that process at devaluation times. We proceed to compute the term (3.6b) for finite maturities under the different assumptions.

\section{Assumption 1: No resetting}

If the expected rate of devaluation is unaffected by a devaluation, integration of the numerator in (3.6b) is easy:

$$
E\left[\int_{0}^{\tau} g(t) d t \mid g(0)=g\right]=\int_{0}^{\tau}\left(g+\mu_{g} t\right) d t=g \tau+\frac{1}{2} \mu_{g} \tau^{2},
$$

and, letting $D_{i}(g ; \tau)$ denote $D(g ; \tau)$ under Assumption $i$, we have that the expected devaluation per unit maturity fulfills

$$
D_{1}(g ; \tau)=g+\mu_{g} \tau / 2
$$

under Assumption 1. While the term $\tilde{\delta}(h ; \tau)$ in $(3.5)$ is less responsive to $h$ and $\tilde{x}$ for longer maturities, the term $D_{1}(g ; \tau)$ in $(3.5)$ is equally responsible to $g$ for all maturities. Thus, for longer maturities the effect of $g$ on the interest rate differential dominates. If $g$ and $\bar{x}$ are positively correlated (which is the case unless $\tilde{f}$ and $g$ are strongly negatively correlated), it follows that for longer maturities we should see a positive correlation between exchange rates and interest rate differentials. If the expected rate of devaluation is expected to increase over time $\left(\mu_{g}>0\right)$, then the differential yield curve will to be upward sloping for sufficiently large maturities, since $\tilde{\delta}(h ; \tau)$ approaches zero for long maturities. Over time, the differential yield curve will be nonstationary: a positive drift in $g$ under Assumption 1 would awkwardly imply that the differential yield curve is on average shifted up at a constant rate with the passage of time.

\section{Resetting of the expected rate of devaluation and of its drift}

Under assumptions 2 and 3 , the expected rate of devaluation tends to revert towards $\bar{g}$ and interest rate differentials are stationary. Further, the expected rate of devaluation is expected to remain constant at $\bar{g}$ after the next devaluation, since the drift $\mu_{g}$ is reset after each devaluation to a value drawn from a distribution with mean zero: this makes it fairly easy to compute the second term in (3.5). Let $T>0$ be the time of the first devaluation after time 0 . The expected rate of devaluation at time $t$ can then be written

$$
E[g(t) \mid g(0)=g]=\left(g+\mu_{g} t\right) \operatorname{Prob}\{T>t \mid g(0)=g\}+\bar{g} \operatorname{Prob}\{T \leq t \mid g(0)=g\} .
$$


We thus need to compute the probability that the first devaluation occurs after a given time $t$, under Assumptions 2 and 3 in turn.

Assumption 2: Constant mean devaluation size

Under Assumption 2 the expected size of the devaluation $\bar{z}$ is constant. Thus, the probability intensity $\nu(t)=g(t) / \bar{z}$ is a Brownian motion with drift $\mu_{g} / \bar{z}$, and we show in the Appendix that in this case

$$
\operatorname{Prob}\{T>t \mid g(0)=g\}=e^{-\left(g t+\mu t^{2} / 2\right) / z} .
$$

Using (3.10) in (3.9) and integrating (3.6b) we obtain, after some algebra:

$$
\begin{aligned}
& D_{2}(g ; \tau)=\frac{\bar{z}\left(1-e^{-\left(g r+\mu_{0} r^{2} / 2\right) / \bar{z}}\right)+G}{\tau}, \text { with } \\
& G= \begin{cases}\bar{g}\left(\tau-\frac{\bar{g}}{g}\left(1-e^{-g \tau / \bar{z}}\right)\right) & \text { if } \mu_{g}=0, \\
\left.\bar{g}\left(\tau-\frac{\sqrt{\pi}}{2} \sqrt{\frac{2 \bar{\xi}}{\mu_{\theta}}} e^{\frac{g^{2}}{2 \mu_{g} g^{z}}}\left(\operatorname{Erf}\left(\sqrt{\frac{\mu_{Q}}{2 \bar{z}}}\left(\tau+\frac{g}{\mu_{\theta}}\right)\right)-\operatorname{Erf}\left(\sqrt{\frac{\mu_{Q}}{2 \bar{z}}} \frac{g}{\mu_{g}}\right)\right)\right)\right) & \text { if } \mu_{g} \neq 0,\end{cases}
\end{aligned}
$$

where $\operatorname{Erf}(x)$ denotes the so called error function, $\operatorname{Er} f(x)=(2 / \sqrt{\pi}) \int_{0}^{x} e^{-t^{2}} d t$.

Assumption 9: Constant probability intensity

Here, the probability intensity $\nu$ is a constant $\bar{\nu}$, while the mean size of the devaluation $\bar{z}(t)=g(t) / \bar{\nu}$ is a Brownian motion. Then,

$$
\operatorname{Prob}\{T>t \mid g(0)=g=\bar{z} \bar{\nu}\}=e^{-\bar{\nu} t}
$$

Using this in (3.9) and (3.6b), we obtain

$$
D_{3}(g ; \tau)=\frac{1}{\tau}\left(\left(\frac{g}{\bar{\nu}}+\frac{\mu_{g}}{\bar{\nu}^{2}}\right)\left(1-e^{-\bar{\nu} r}\right)-\frac{\mu_{g} \tau}{\bar{\nu}} e^{-\bar{\nu} r}+\left(\nu \tau-\left(1-e^{-\bar{\nu} \tau}\right)\right) \overline{\bar{z}}\right),
$$

where we recall that $\bar{z}=\bar{g} / \bar{\nu}$.

For zero drift in $g$, we see that Assumption 2 and 3 give identical differential yield curves. Figure 3.4 shows the functions $D_{i}(g ; \tau), i=1,2,3$, plotted against $\tau$ for $g=3$ percent per year, $\mu_{g}=1$ percent per year squared, $\bar{g}=2$ percent per year, $\bar{z}=10$ percent (under Assumption 2), $\bar{\nu}=20$ percent per year and $\overline{\bar{z}}=\bar{g} / \bar{\nu}=10$ percent (under Assumption 3). We note that both $D_{2}(g ; \tau)$ and $D_{2}(g ; \tau)$ approach $\bar{g}$ as maturity goes to infinity, and that convergence is much faster under Assumption 2. Given a positive drift. 
Assumption 3 results in a differential yield curve above that resulting from Assumption 2 when the random $\nu(0)=g(0) / \bar{z}$ under Assumption 2 coincides with the constant $\bar{\nu}=$ $g(0) / \bar{z}(0)$ under Assumption 3. And, for positive $g$, Assumption 2 or 3 imply that the term $D(g ; r)$ may have a hump shape if $\mu_{g}$ is sufficiently positive.

The differential yield curve is then given by the vertical addition of the relevant curve in Figure 3.3, resulting from expected depreciation per unit maturity within the band, to the curve for each assumption in Figure 3.4, resulting from the expected depreciation per unit maturity caused by devaluation. The resulting relationship can clearly have a variety of shapes, depending upon the interaction between its two components.

With regard to the correlation between interest rate differentials and the exchange rate, we have seen that the sensitivity of the interest rate differentials to the exchange rate for given levels of $g$ decreases with increased maturity. The sensitivity of the interest rate differential to $g$ for given levels of the exchange rate is high for short maturities, may evcn increase in the maturity if $\mu_{g}$ is positive, but under Assumption 2 or 3 eventually decreases and flattens out for long maturities. If the exchange rate is positively correlated with $g$, it is possible that the correlation between interest rate differentials and exchange rates is negative or small positive for short maturities, positive for intermediate maturities, and close to zero for long maturities.

\section{Empirical implications}

In light of the evidence collected by Flood, Rose and Mathieson (1990) and by the other contributions reviewed in the Introduction, empirically successful models of exchange rate determination should allow for (at least) two less than perfectly correlated sources of time variation in exchange rates and interest rate differentials. Of course, a second state variable could be introduced in target zone models in a variety of ways: but we can argue that stochastic devaluation risk is empirically and theoretically preferable to most alternatives.

A time-varying risk premium (as in Froot and Obstfeld (1989)) would introduce a variable wedge between interest rate differentials and depreciation rates, and would be the most straightforward way to introduce noise in the relationship between interest rate differentials and exchange rates predicted by simple target zone models: absent any devaluations, the solution would formally be the same as the one we have derived here. It is unlikely, however, that stochastic risk would provide a complete explanation of the available evidence. At a theoretical level, risk premia are likely to be small and any fluctuations in them should not matter much (Svensson (1990a). Empirically, devaluations do indeed occur, and interest rate differentials appear to be correlated with evidence of perceived 
devaluation risks, such as discussions of imminent realignments in news media.

Less straightforwardly, a second state variable could be introduced allowing the parameters of the $f$ process to vary over time. If such variability were less than perfectly related to the exchange rate's position in the band, it would indeed affect the expected depreciation rate for given level of $f$, and could explain the disappointing empirical fit of simpler target-zone models. However, the theoretical basis for this extension would be hard to specify and, empirically, there would be some presumption that looking at shorter sample the theoretical nonlinear relationship should be more apparent, but this is not the case in Flood, Rose and Mathieson (1990).

This leaves stochastic fluctuations in expected devaluation rates as a realistic and tractable source of noise in the empirical relationship between interest rate differentials and exchange rate deviations from central parities. In what follows, we show that our model indeed appears capable of rationalizing existing evidence on exchange rates and interest rate differentials, and we suggest ways in which our model might be empirically implemented.

\section{Simulation experiments}

In Figures 4.1-4.3 we report simulated exchange rates and instantaneous interest rates differentials, computed weekly for a period of nine years. ${ }^{11}$ All simulations use the common parameter values $\alpha=1$ year, $\sigma=5$ percent per year ${ }^{1 / 2}, \mu_{f}=1$ percent per year, $\mu_{g}=1$ percent per year squared (which results in $\mu=\mu_{f}+\alpha \mu_{s}=2$ percent per year), and $\rho=0$. The exchange rate band is \pm 1.5 percent, and the $(\log )$ exchange rate starts out in the interior of the first band (corresponding to $h$ starting out at zero). All simulations use the same seeds to generate sequences of normal increments and of uniformly distributed random variables (which trigger devaluations), and differ only in the relative importance of the two sources of exchange rate fluctuation, $\tilde{f}$ and $g$.

The simulations are done under a variant of Assumption 2. The devaluation size is nonstochastic and fixed at $\bar{z}=10$ percent, and the probability intensity $\nu=g / \bar{z}$ is stochastic. The expected rate of devaluation starts out at $g=1$ percent per year, corresponding to a probability intensity of $\nu=10$ percent per year, and is reset to the same value when a devaluation occurs. In contrast to Assumption 2, the drift $\mu_{g}$ is not reset but remains

11 The simulations use the simple formulae valid for instantaneous interest rate differentials. Although a week should be treated as a discrete time interval, the model's predictions are almost exactly the same for an infinitesimal time increment as for $\Delta t=1 / 52$. 
at 1 percent per year squared, which is realistic for a structurally weak currency. Also in contrast to what is assumed during the discussion of finite maturity interest rate differentials, the exchange rate is set at the strong edge of the new band after a devaluation and at the weak edge of the new band after a revaluation; since the jump in the exchange rate is exactly $\bar{z}$, this means that the jump in the central parity will usually not be exactly $\bar{z}$.

Figures 4.1a-c show simulations for the case when the relative variability of the expected rate of devaluation is low: $\sigma_{g}=1$ percent per year ${ }^{3 / 2}$ versus $\sigma_{f}=4.9$ percent per year ${ }^{1 / 2} .12$ Figure $4.1 \mathrm{a}$ shows a plot of the exchange rate during the first nine years, during which three devaluations occur. The probability intensity of devaluations is almost constant, and devaluations can and do occur when the exchange rate is away from the weak edge of its band. Each of the dots in Figure 4.1b (which should be compared to Figure 3.1) corresponds to a simulated observation of exchange rate deviations from central parity, $\tilde{x}$, and interest rate differentials. The solid curve is the expected depreciation within the band, $\delta(\tilde{x} ; 0)$, and the expected rate of devaluation for each observation is the vertical distance between the observation and the solid curve. As predicted by the model, interest rate differentials and exchange rates are negatively correlated in the simulated sample. Figure 4.1c shows a plot of the exchange rate and $\tilde{f}$, and should be compared to Figure 2.2. The solid curve shows the function $\tilde{x}(h)$ with $h$ equal to $\tilde{f}$ (which occurs for $g$ equal to zero). The observations can be understood as being generated by points on curves shifted left by $\alpha g$. Since $g$ is fairly stable, there is a positive correlation between the exchange rate and $\tilde{f}$.

Figures $4.2 \mathrm{a}-\mathrm{c}$ show simulations for the case when the relative variability of the expected rate of devaluation is about equal to that of $\bar{f}: \sigma_{g}=3.5$ percent per year ${ }^{3 / 2}$ versus $\sigma_{f}=3.6$ percent per year ${ }^{1 / 2}$. In Figure $4.2 \mathrm{a}$, four devaluations occur during the first nine ycars; since the probability intensity $g$ of devaluations is now variable, and the exchange rate tends to be weak for large $g$ (which is associated to large $h$ ), devaluations tend to occur when the exchange rate is close to the weak edge of its band. In Figure $4.2 \mathrm{~b}$, there is hardly any correlation between exchange rates and interest rate differentials, and the relationship between the exchange rate and $\tilde{f}$ is quite weak (though still positive) in Figure $4.2 \mathrm{c}$.

Figures $4.3 \mathrm{a}-\mathrm{c}$ show simulations for the case when the relative variability of the ex-

12 This results in $\sigma=\sqrt{\sigma_{f}^{2}+\alpha^{2} \sigma_{g}^{2}}=5$ percent per year ${ }^{1 / 2}$. Since the dimension of $g$ is $1 /$ time, the dimension of $\sigma_{g}$ is actually $1 /(\text { time })^{3 / 2}$. The relative variability between $g$ and $\tilde{f}$ is measured by $\alpha \sigma_{g} / \sigma_{f}$, and we use $\sigma_{g} / \sigma_{f}$ since $\alpha=1$ year in the simulations. 
pected rate of devaluation is high: $\sigma_{g}=4.9$ percent per year ${ }^{3 / 2}$ versus $\sigma_{f}=1$ percent per year ${ }^{1 / 2}$. In Figure 4.3a, four devaluations occur during the first nine years, and the exchange rate is close to the weak edge of its band when devaluations occur. In Figure $4.3 \mathrm{~b}$, the correlation between exchange rates and interest rate differentials is positive, and in Figure $4.3 \mathrm{c}$ there is hardly any relationship between the exchange rate and $\tilde{f}$.

\section{Inferring devaluation risk from target zone data}

In our model, exchange rates and interest rate differentials are endogenous and jointly determined by fluctuations in (exogenous) fundamentals and devaluation risks. The parameters of the model could be estimated in a variety of ways: $:^{13}$ here, we shall only sketch how the behavior over time of devaluation risk could be inferred from data on interest and exchange rate, leaving it to future research to provide a more rigorous treatment of econometric problems and to carry out actual empirical work.

If uncovered interest parity holds, the expected rate of depreciation $E_{t}[d x] / d t$ can be measured by (observable) short-term interest rate differentials: by equation (3.3), then,

$$
E_{\mathrm{t}}[d x] / d t=i(t)-i^{*}(t)=E_{\mathrm{t}}[d \bar{x}] / d t+g(t)
$$

where $i$ and $i^{*}$ denote the interest yield for instantaneously-maturing bonds denominated in (respectively) home and foreign currencies. Thus,

$$
g(t)=i(t)-i^{*}(t)-E_{t}[d \tilde{x}] / d t,
$$

and the relative importance of fluctuations in devaluation risk and their stochastic properties can be evaluated examining the difference between interest rate differentials and an estimate of $E_{t}[d \tilde{x}] / d t$, the expected rate of depreciation within the band, at the same point in time. It appears possible to obtain such an estimate from a series of exchange rate observations: in our model, the process of exchange rate deviations from central parities has the stochastic differential

$$
d \tilde{x}(t)=\tilde{\delta}(t) d t+\sigma_{\mathbf{z}}(t) d W(t)
$$

where the drift $\tilde{\delta}(t)$ and the standard deviation $\sigma_{x}(t)$, while stochastic, are uniquely determined by the state variable $h(t)$ and (hence) by the exchange rate within the band at every point in time. Integrating (5.2) over finite time intervals of length $\Delta t$ (corresponding

13 See Pessach and Razin (1990), Smith and Spencer (1990) and Flood, Rose and Mathieson (1990). 
to the frequency at which exchange rate observations are available), it may be possible to obtain an estimable equation: still, the dependence of $\tilde{\delta}(t)$ on $\tilde{\boldsymbol{x}}(t)$ is highly nonlinear and has no closed form; over finite time intervals, the expected depreciation rate has the infinite-series representation derived by Svensson (1990b). Thus, exact estimation of expected depreciation within the band is exceedingly complex, and nonparametric methods may need to be used.

Svensson (1990b), however, shows that the expected rate of depreciation over a finite time interval equal to a month or longer may be a fairly linear function of $\tilde{x}$ for reasonable parameter values (see Figure 3.2 above). Thus, it may be an acceptable approximation to write

$$
\Delta \tilde{x}(t) / \Delta t=\beta_{0}+\beta_{1} \tilde{x}(t)+\epsilon(t)
$$

where the error term $\epsilon(t)=\int_{t}^{t+\Delta t} \sigma_{z}(s) d W(s) / \Delta t$ is uncorrelated with $\tilde{x}(t)$ (by rational expectations), heteroskedastic, and serially independent for non-overlapping time intervals. Standard econometric methods (see e.g. Hodrick (1987)) could then be used to obtain consistent estimates of $\beta_{0}$ and $\beta_{1}$ from a series of exchange rate deviations from central parities. ${ }^{14}$ Further, similar equations would be appropriate for a wide variety of intervention rules and underlying fundamental disturbances. In many models, the drift of $\tilde{x}(t)$ would still depend only on $\tilde{x}(t)$, and equation (5.3) (or nonlinear modifications of it) could still be used to estimate the expected within-band depreciation rate. In models with more state variables (e.g. reserves), similar methods would be applicable if data on the other determinants of exchange rate dynamics are available.

The expected rates of devaluation could then be computed by subtracting expected depreciation with the band from the corresponding interest rate differentials, and it would be interesting to relate the series thus obtained to competitiveness, reserves, political credibility, and other potential determinants of devaluations.

\section{Conclusions}

This paper shows that the incorporation of stochastic devaluation risk in target zone models results in a rather rich data-generation structure, which may explain the poor empirical fit

14 The exchange rate's position in the band may well change at a devaluation (then, the expected devaluation size reflected in interest rate differentials is the sum of the jump in the central parity and of the jump of the exchange rate within the band). Thus, the observations at which a devaluation occurs include an $\tilde{x}$ jump as well as an $x$ jump, and should be excluded from the sample used to estimate the drift of continuous, within-band exchange rate fluctuations. 
of models without stochastic devaluation risk and provide an interesting interpretation for the variability those models leave unexplained. It remains to see, after careful empirical work, whether the model with stochastic devaluation risk will actually better fit the data.

We would immediately like to add that we do not, of course, believe that the model we have developed here is all there is needed to explain the data. For instance, in the model we have developed, the unconditional distribution of exchange rates within the band is still $U$-shaped, which does not fit the data. But we do believe that separate stochastic devaluation risk is an essential ingredient in real world target zone exchange rate regimes, and that it is important to take these devaluation risks into account in the modeling of target zones. We therefore believe that it would be useful to incorporate stochastic devaluation risk in the existing and future target zone models which try to improve their empirical performance by considering implicit bands within the official bands (Klein (1990)), intramarginal intervention, increased devaluation risk at the edges of the band (Miller and Weller (1989) and Bertola and Caballero (1990b)), reserves (Bertola and Caballero (1990a) and Dumas and Delgado (1990)), sticky prices (Miller and Weller (1989)), jumps in fundamentals (Perraudin (1990)), etc. A particularly challenging task is that of developing theoretical models where devaluations and devaluation risk are the endogenous outcome of political and economic interactions. Empirical work aimed at extracting expected rates of devaluation from exchange rates and from in terest rate differentials for different maturities should provide useful input for such theoretical models. 


\section{Appendix: Derivation of (3.10)}

Let $P(t)=\operatorname{Prob}\{T>t \mid g(0)=\bar{z} \nu(0)=g=\bar{z} \nu\}=\operatorname{Prob}\{T \notin[0, t] \mid \nu(0)=\nu\}$. Then note that

$$
\begin{aligned}
P(t+d t) & =P(t)+P^{\prime}(t) d t+o(d t) \\
& =\operatorname{Prob}\{T \notin[0, t+d t] \mid \nu\} \\
& =\operatorname{Prob}\{T \notin[0, t] \mid \nu\} \operatorname{Prob}\{T \notin(t, t+d t] \mid \nu\} \\
& =P(t)\{1-E[\nu(t) \mid \nu(0)=\nu] d t+o(d t)\}
\end{aligned}
$$

For $d t \rightarrow 0$, this yields

$$
P^{\prime}(t)=-P(t) E[\nu(t) \mid \nu(0)=\nu],
$$

Noting that $E[\nu(s) \mid \nu(0)=\nu]=\left(g+\mu_{g} s\right) / \bar{z}$, integrating, and using $P(0)=1$, we obtain

$$
P(t)=e^{-\int_{0}^{t} E[\nu(s) \mid \nu(0)=\nu] d s}=e^{-\left(g t+\mu_{\theta} t^{2} / 2\right) / \bar{z}} .
$$




\section{REFERENCES}

Bertola, Giuseppe, and Ricardo J. Caballero (1990a), "Sustainable Intervention Policies and Exchange Rate Dynamics," Working Paper, Columbia and IER; to appear in P.Krugman and M.Miller (eds.), Exchange Rate Targets and Currency Bands, London: C.E.P.R. (forthcoming).

Bertola, Giuseppe, and Ricardo J. Caballero (1990b), "Target Zones and Realignments," C.E.P.R. Discussion Paper \#398.

Delgado, Francisco, and Bernard Dumas (1990), "Monetary Contracting between Central Banks and the Design of Sustainable Exchange-Rate Zones," Working Paper, Wharton School, University of Pennsylvania.

Flood, Robert P., Andrew K. Rose, and Donald J. Mathieson (1990), "Is the EMS the Perfect Fix? An Empirical Exploration of Exchange Rate Target Zones," International Finance Discussion Paper \#388, Board of Governors of the Federal Reserve System.

Froot, Kenneth A., and Maurice Obstfeld (1989), "Exchange Rate Dynamics Under Stochastic Regime Shifts: A Unified Approach," Working Paper, N.B.E.R.

Hodrick, Robert J. (1987), The Empirical Evidence on the Efficiency of Forward and Futures Foreign Exchange Markets, London: Harwood.

Klein, Michael W. (1990), "Big Effects of Small Interventions: The Informational Role of Intervention in Exchange Rate Policy," Working Paper, Clark University.

Krugman, Paul (1990), “Target Zones and Exchange Rate Dynamics," Quarterly Journal of Economics , (forthcoming).

Lehmussaari, Olli-Pekka, and Antti Suvanto (1990), "The Finnish Experience of Maintaining a Currency Band in the 1980s," Working Paper, Bank of Finland.

Meese, Richard A., and Andrew K. Rose (1990), "Nonlinear, Nonparametric, Nonessential Exchange Rate Estimation," American Economic Review 80-2, 192-196.

Miller, Marcus, and Paul Weller (1989), "Exchange Rate Bands and Realignments in a Stationary Stochastic Setting," in M. Miller, B. Eichengreen, and R. Portes (eds.), Blueprints for Exchange Rate Management, New York: Academic Press.

Perraudin, William R. M. (1990), "Exchange Rate Bands with Point Process Fundamentals," Working Paper, International Monetary Fund.

Pessach, Shula, and Assaf Razin (1990), "Targeting the Exchange Rate: An Empirical Investigation," Working Paper, International Monetary Fund. 
Smith, Gregor W., and Michael G. Spencer (1990), "Estimation and Testing in Models of Exchange-Rate Target Zones and Process Switching," Working Paper.

Svensson, Lars E. O. (1989), “Target Zones and Interest Rate Variability," Seminar Paper \# 457, Institute of International Economic Studies, University of Stockholm.

Svensson, Lars E. O. (1990a), "The Foreign Exchange Risk Premium in a Target Zone with Devaluation Risk," Seminar Paper \# 475, Institute of International Economic Studies, University of Stockholm.

Svensson, Lars E. O. (1990b), "The Term Structure of Interest Rates in a Target Zone: Theory and Swedish Data," Working Paper, Institute of International Economic Studies, University of Stockholm. 

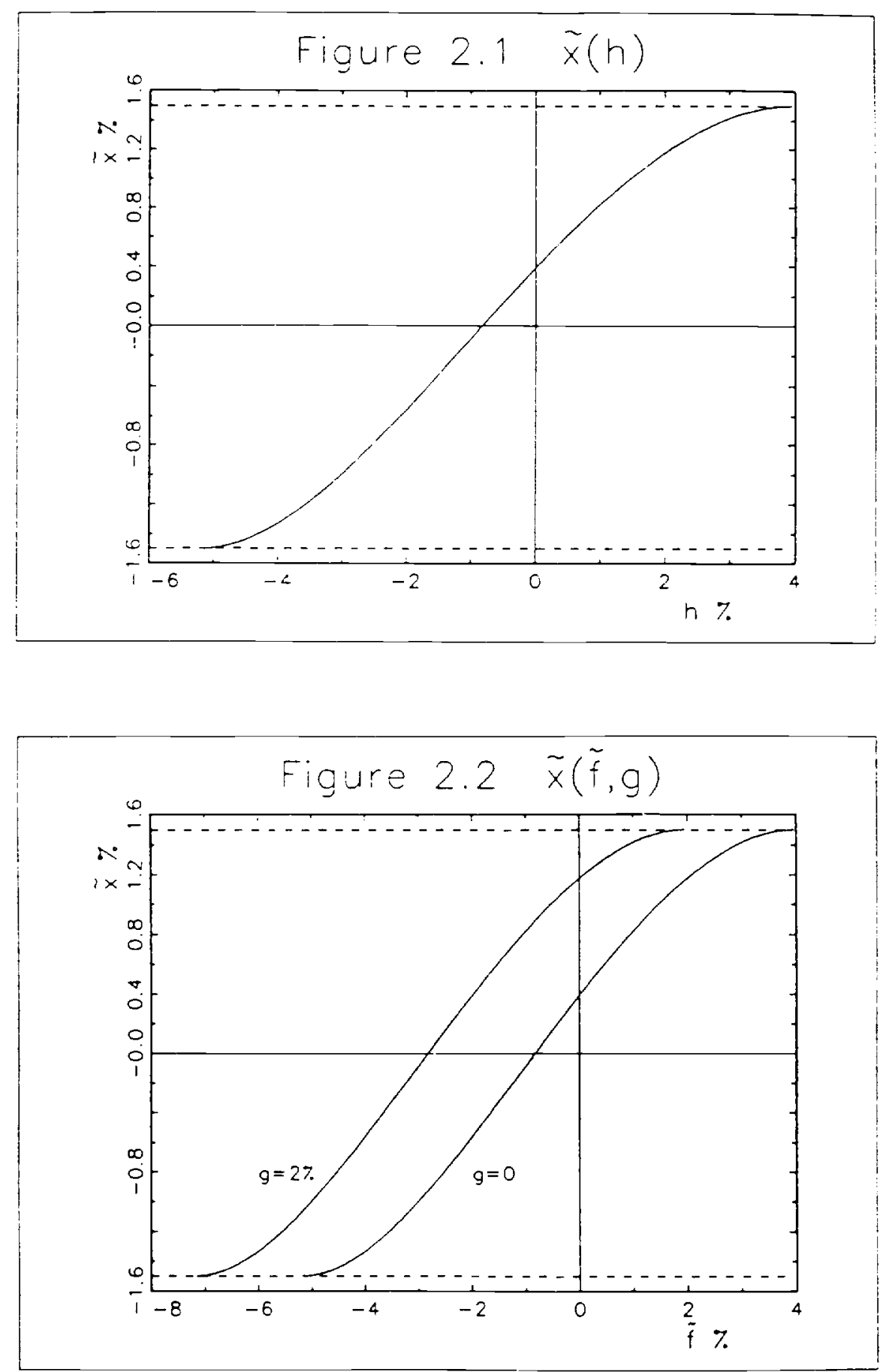


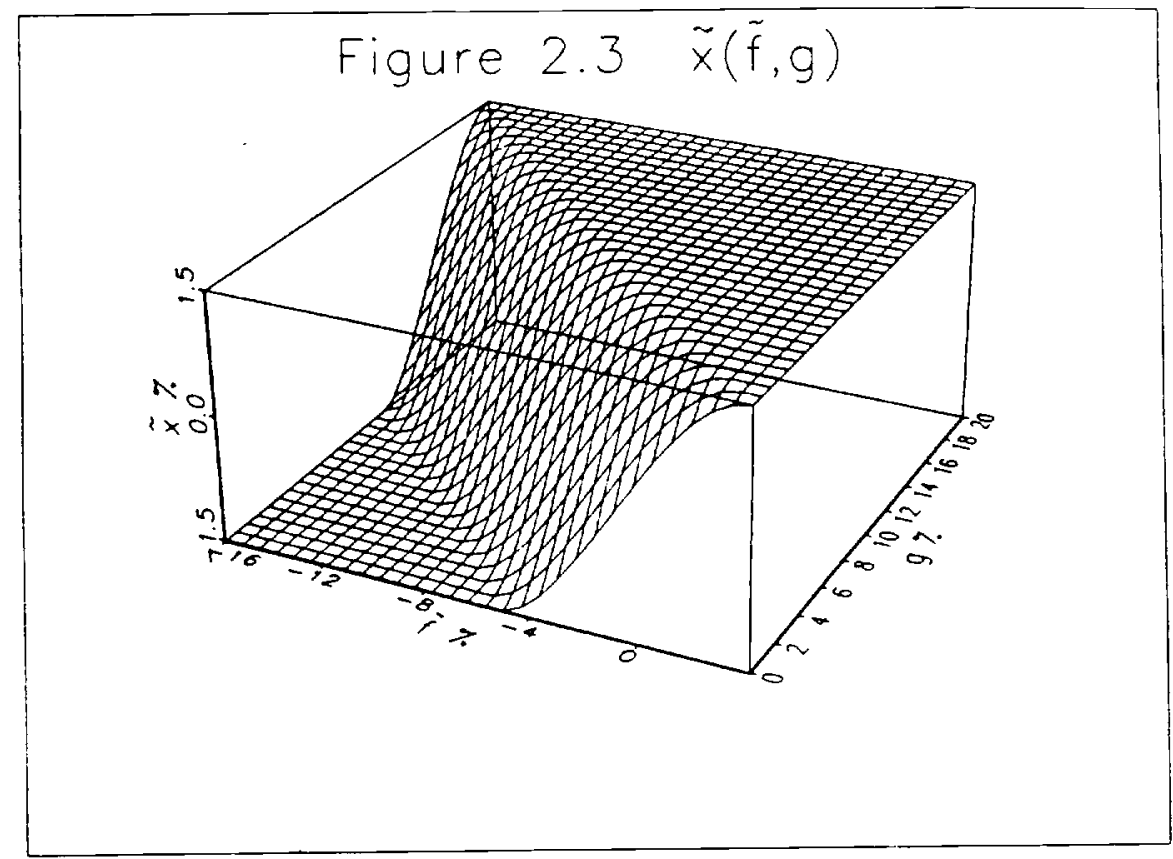

Figure $3.1 \tilde{\delta}(\tilde{x} ; 0)$ and $\delta(\tilde{x}, g ; 0)$

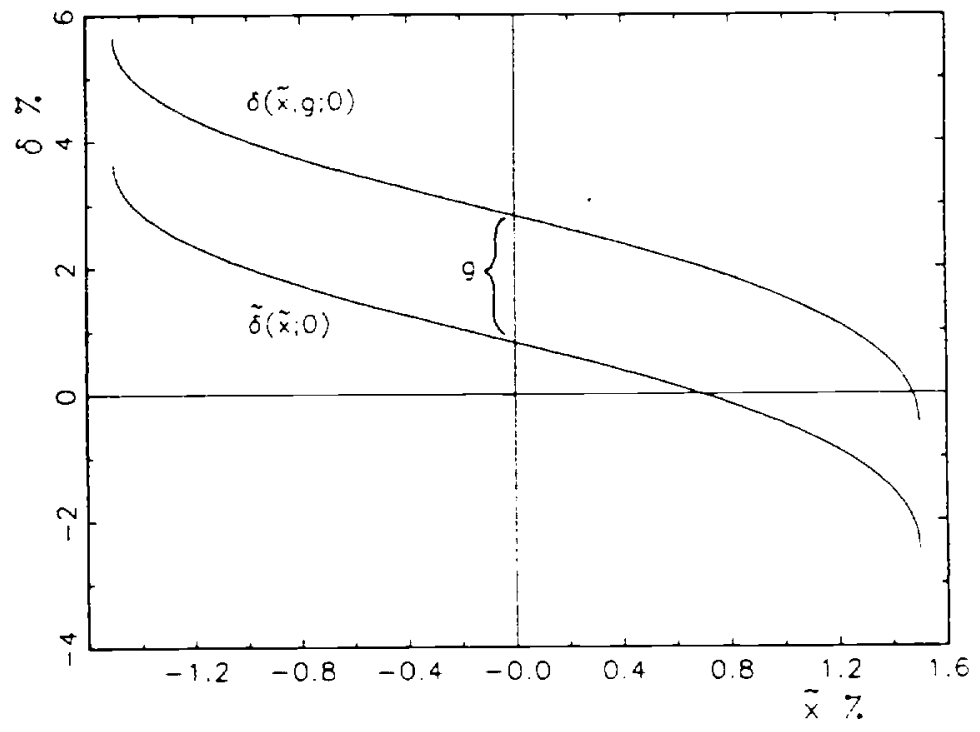



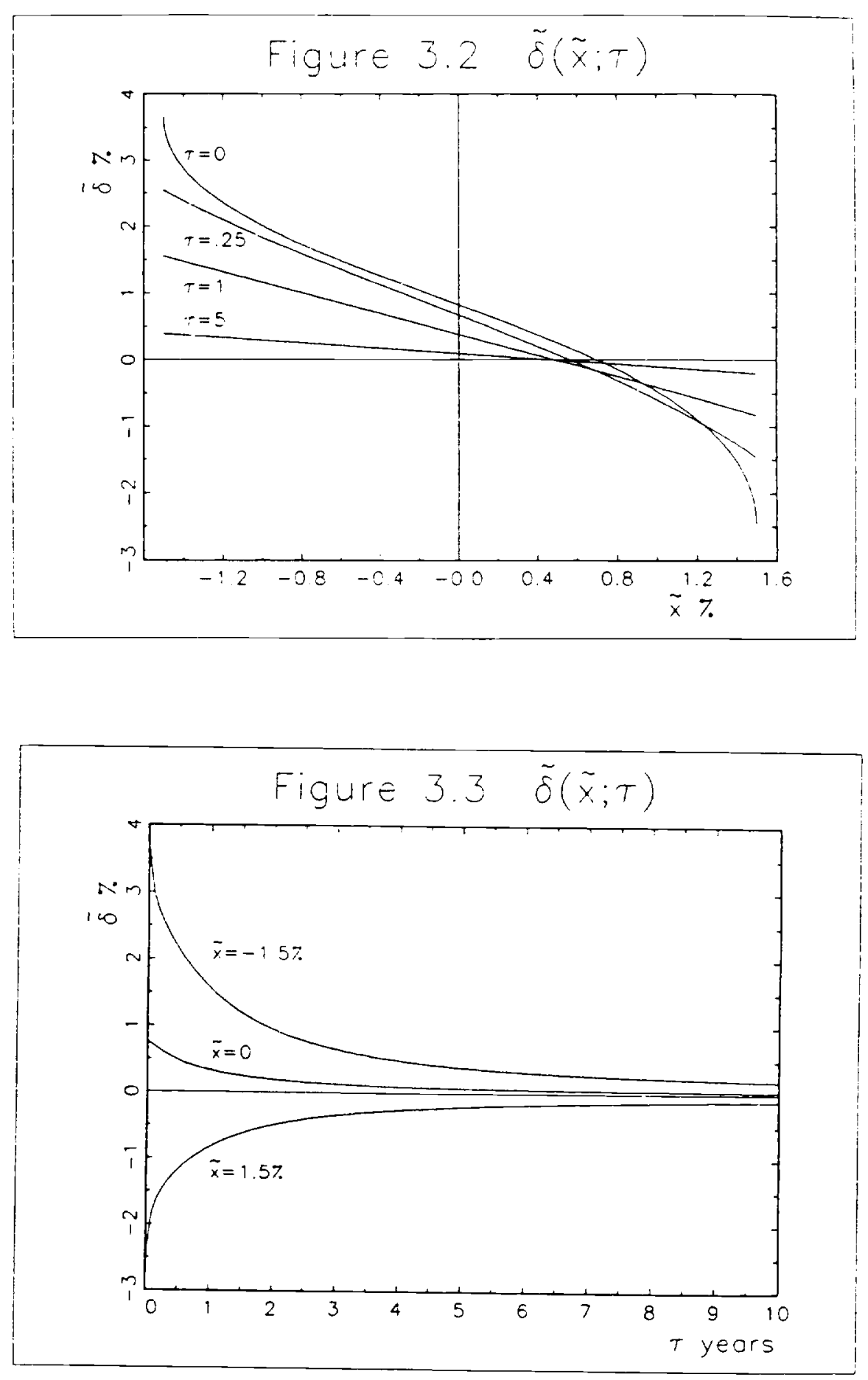

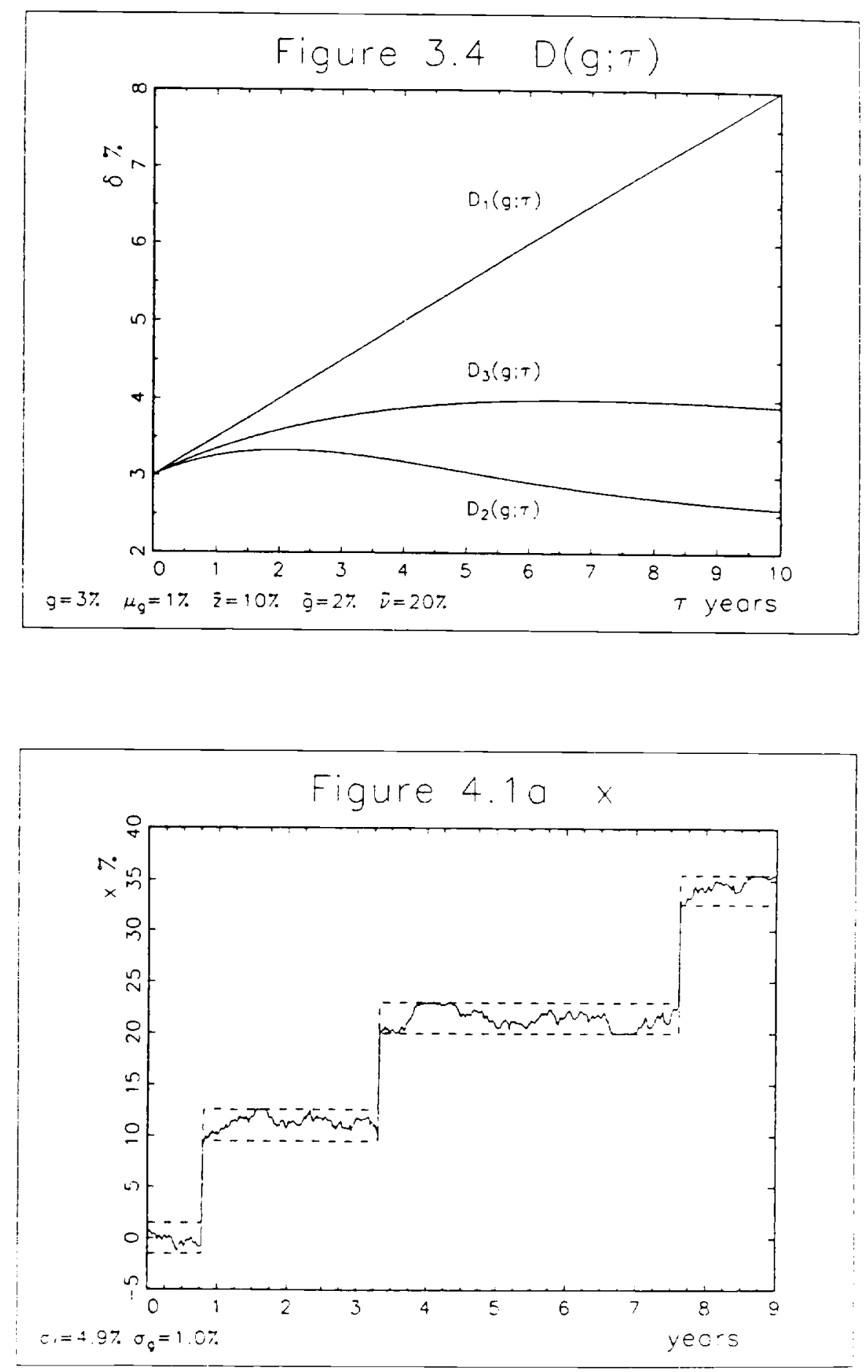



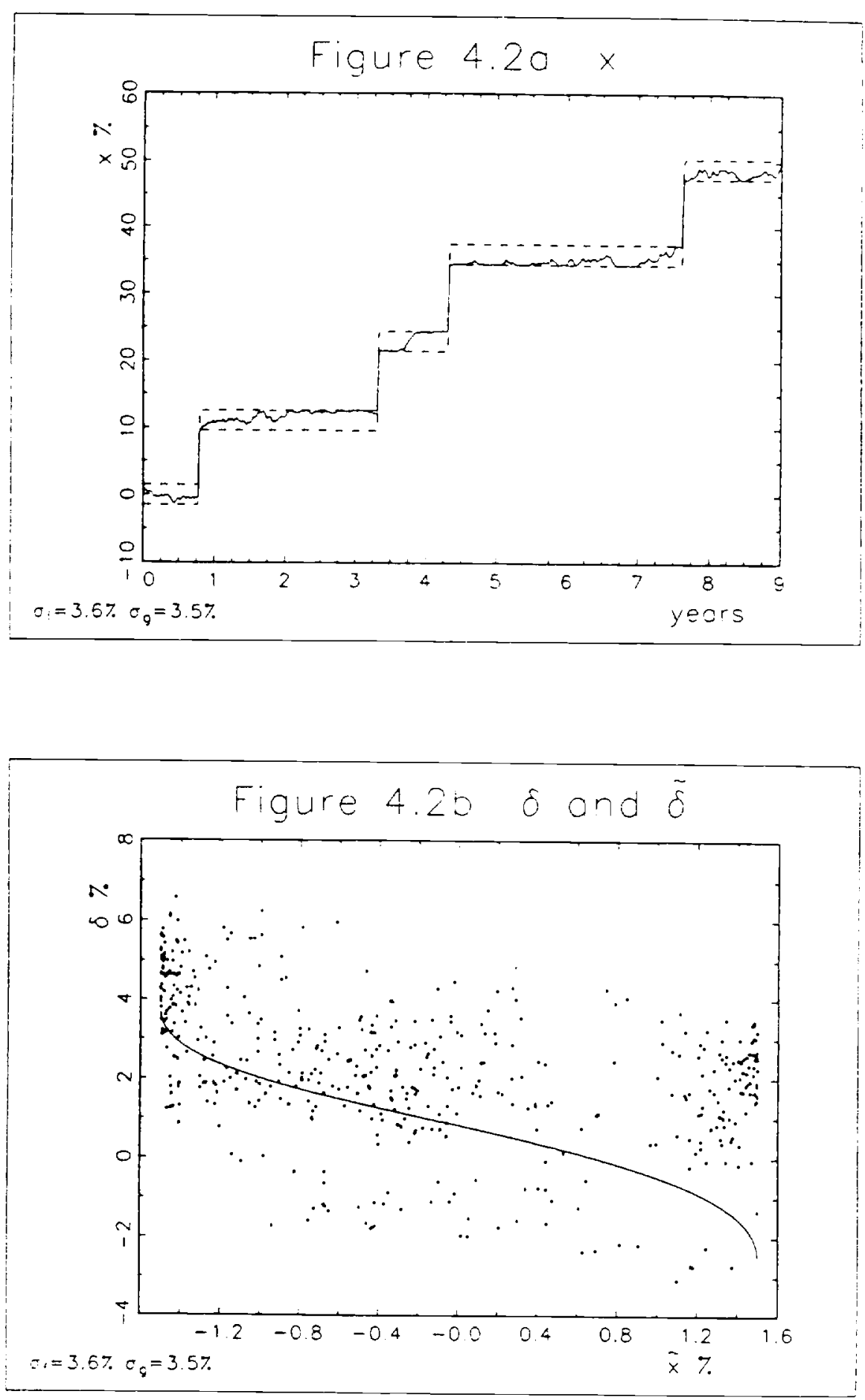

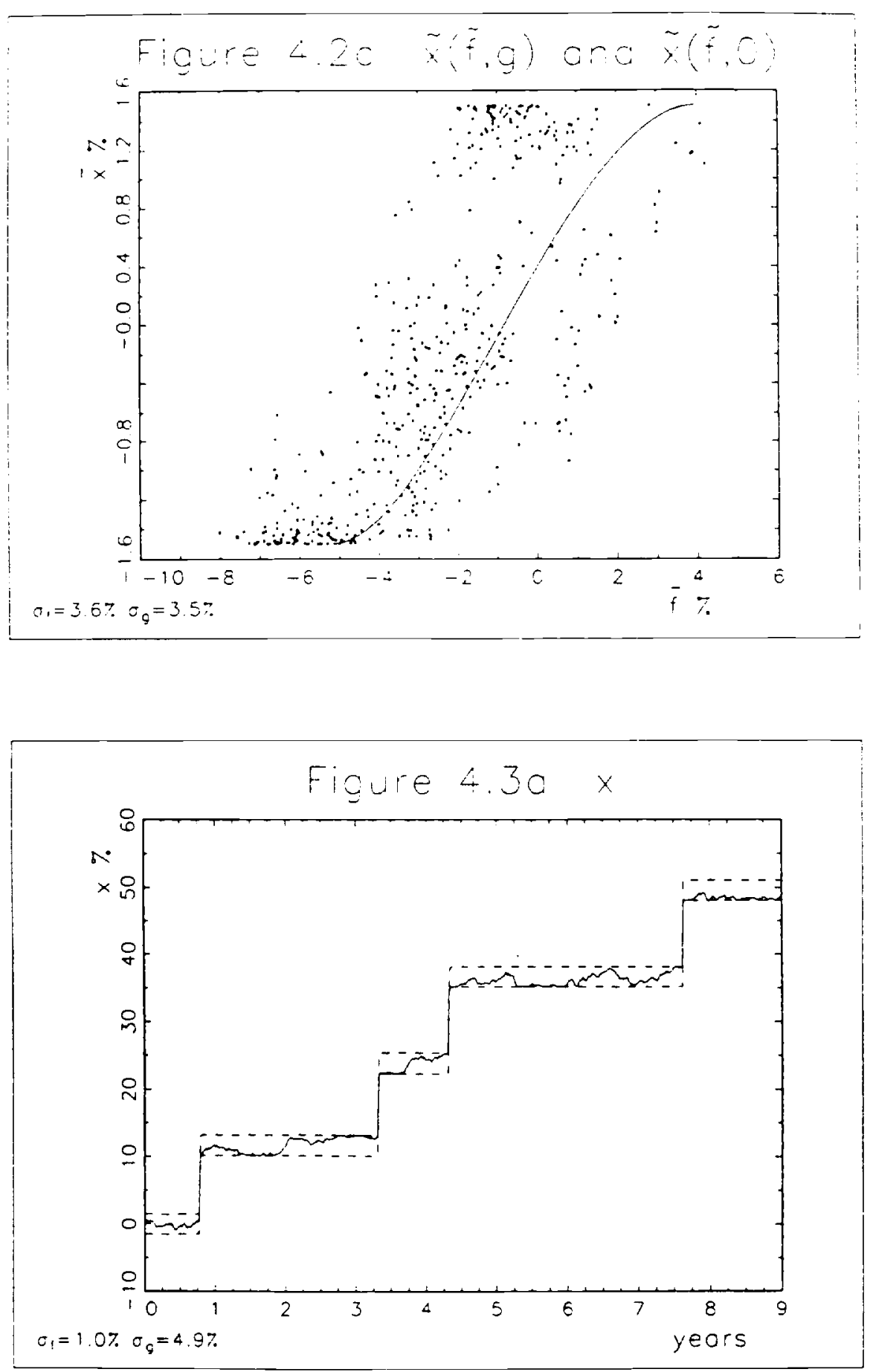

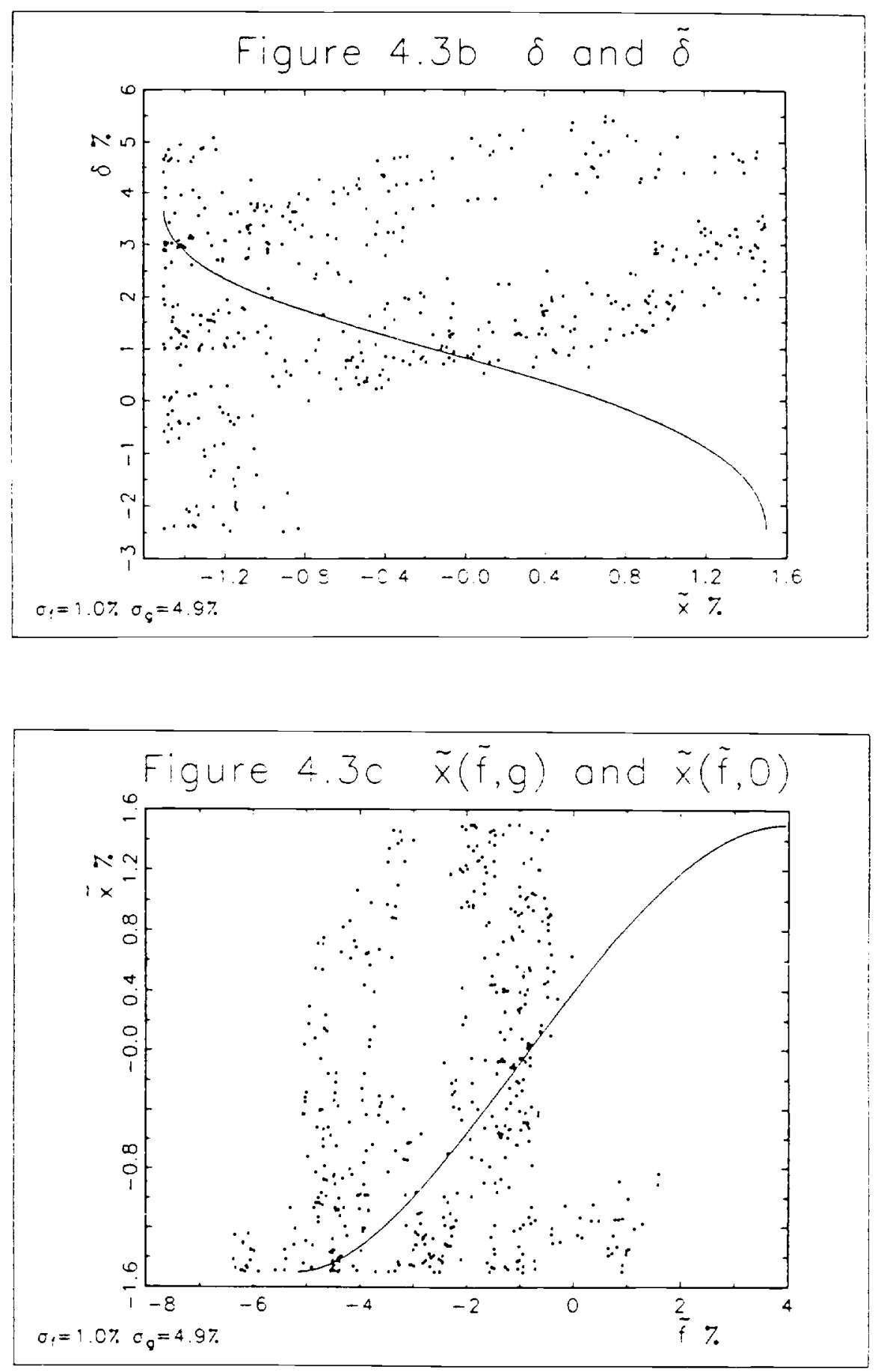